\title{
Marcadores salivares para a periodontite crônica no diabético tipo 2
}

\author{
Salivar markers for chronic periodontitis in diabetic type 2
}

\begin{abstract}
RESUMO
Introdução: a periodontite crônica é a sexta doença infecciosa mais prevalente no mundo, seus fatores de risco são o aumento da idade, tabaco, fatores genéticos, obesidade e distúrbios sistêmicos como o diabetes. O diabetes é uma pandemia tanto em países desenvolvidos como em desenvolvimento, é uma doença complexa com graus variados de complicações sistêmicas e orais. Assim, é urgente a necessidade de um método fácil para detectá-la permitindo a intervenção antes da progressão da doença periodontal. Á análise de metabólitos da saliva tem sido proposta como uma ferramenta efetiva para o diagnóstico e tratamento periodontal.

Objetivo: discorrer sobre alternativas de diagnóstico de periodontite crônica em pacientes diabéticos tipo 2 utilizando marcadores salivares.

Metodologia: Foi realizada uma busca eletrônica no Portal de Periódicos da Coordenação de Aperfeiçoamento de Pessoal de Nível Superior (CAPES), para a realização do referido levantamento, utilizou-se os descritores "Type 2 diabetes mellitus, chronic periodontitis, Biomarkers, Saliva".

Resultados: a detecção precoce da periodontite crônica não é apenas vital para reduzir a sua gravidade e prevenir complicações, mas também crítico para aumentar a taxa de sucesso da terapia. Conclusão: a utilização de biomarcadores na medicina é uma realidade difundida e tem relação direta com a modernização dos meios de saúde, na odontologia vem se pleiteando seu uso, mas ainda necessita de maiores estudos e aperfeiçoamentos das técnicas já empregadas com o intuito de diminuir seus custos, aumentar especificidade e acesso dos profissionais de saúde.
\end{abstract}

Palavras chave: Biomarcadores, Diabetes Mellitus Tipo 2, Periodontite Crônica, Saliva.

\begin{abstract}
Introduction: Chronic periodontitis is the sixth most prevalent infectious disease in the world. Its risk factors are increased age, tobacco, genetic factors, obesity, and systemic disorders such as diabetes. Diabetes is a pandemic in both developed and developing countries, it is a complex disease with varying degrees of systemic and oral complications. Thus, there is an urgent need for an easy method to detect it allowing intervention before the progression of periodontal disease. The analysis of saliva metabolites has been proposed as an effective tool for periodontal diagnosis and treatment.

Objective: To discuss alternatives for diagnosis of chronic periodontitis in type 2 diabetic patients using salivary markers.

Methodology: An electronic search was performed in the Portal of Periodicals of the Coordination of Improvement of Higher Level Personnel (CAPES). The descriptors "Type 2 diabetes mellitus, chronic periodontitis, Biomarkers, Saliva" were used to perform this survey.

Results: early detection of chronic periodontitis is not only vital to reduce its severity and prevent complications, but also critical to increase the success rate of therapy.

Conclusion: the use of biomarkers in medicine is a widespread reality and has a direct relationship with the modernization of health facilities. In dentistry, its use has been sought, but it still needs further studies and improvements of techniques already employed with the intention of reducing its Increase the specificity and access of health professionals.
\end{abstract}

Keywords: Biomarkers, Chronic Periodontitis, Diabetes Mellitus Type 2, Saliva.

\author{
Romário Correia dos Santos* \\ Roberto Carlos Mourão Pinho** \\ Renata Cimões***
}

* Acadêmico do curso de graduação em odontologia da Universidade Federal de Pernambuco

- UFPE, Recife - PE, Brasil.

** Doutorando em clínica integrada - UFPE,

Recife - PE, Brasil.

*** Professora doutora efetiva do Departamento de Prótese e Cirurgia Buco facial - UFPE, Recife - PE, Brasil.

Autor Correspondente:

Roberto Carlos Mourão Pinho

Curso de Odontologia da Universidade Federal de Pernambuco - UFPE

Centro de Ciências da Saúde - CCS I Departamento de Prótese e Cirurgia Buco Facial. Av Prof. Moraes Rego, s/n - Cidade Universitária Recife - PE | 50670-901

Telefone: (81) 21268817 |

E-mail: robertomouraopinho@yahoo.com.br

Submetido em: 6-11-2017

Aceito em: 9-4-2017 


\section{INTRODUÇÃO}

A periodontite crônica é a sexta doença infecciosa mais prevalente no mundo ${ }^{1}$, o seu impacto financeiro mundial é estimado em US \$ 53,99 bilhões ${ }^{2}$. Cerca de 5 a $15 \%$ dos adultos em todo o mundo têm periodontite grave ${ }^{3}$. É uma doença inflamatória crônica que acomete os tecidos adjacentes ao redor do dente e pode resultar em perda irreversível do ligamento periodontal, tecidos conjuntivos, cemento e osso alveolar ${ }^{4}$. O seu início está associado a uma comunidade polimicrobiana anormal, denominada Disbiose ${ }^{5}$.

Os fatores de risco para o estabelecimento e desenvolvimento da doença periodontal incluem aumento da idade, tabaco, álcool, fatores genéticos, obesidade, estresse psicológico e distúrbios sistêmicos como o diabetes ${ }^{6}$. O diabetes é uma pandemia tanto em países desenvolvidos como em desenvolvimento, é uma doença complexa com graus variados de complicações sistêmicas e orais, e a cavidade oral serve como uma fonte contínua de agentes infecciosos que podem agravar ainda mais o estado fisiológico do doente ${ }^{7}$.

Vários mecanismos, incluindo alterações a resposta do hospedeiro e na composição do microbiota subgengival, foram propostas para explicar uma maior susceptibilidade dos sujeitos com DM à degradação periodontal ${ }^{8}$. A periodontite crônica é um desfecho comum do DM2 e sua prevalência está ligada diretamente com o grau de estabilidade glicose-insulina, uma vez que esta é influenciada pelo grau de inflamação acometido pela periodontite crônica implicando na resistência hormônio-receptor ${ }^{9}$. As moléculas pró-inflamatórias liberadas pelos tecidos periodontais doentes podem entrar na circulação para induzir resistência à insulina ${ }^{10}$.

Assim, é urgente a necessidade de um método fácil para detectá-la permitindo a intervenção antes da progressão da doença periodontal. Nesta linha, a análise de metabólitos da saliva tem sido proposta como uma ferramenta efetiva para o diagnóstico e tratamento periodontal ${ }^{2}$. A saliva é secretada pelas glândulas salivares e tem múltiplas funções, limpeza e proteção bucal, é antibacteriana e inclusive com efeitos na digestão. Atualmente, é bem aceita como um conjunto de marcadores biológicos. Uma vez que é uma fonte não-invasiva e segura de diagnóstico e prognóstico de doenças precoces na medicina translacional e medicina de precisão, podendo ser um substituto para exames onde o sangue é coletado ${ }^{11}$.

O objetivo dessa revisão de literatura é discorrer sobre alternativas de diagnóstico de periodontite crônica em pacientes diabéticos tipo 2 utilizando marcadores salivares.

\section{MATERIAL E MÉTODOS}

Foi realizada uma busca eletrônica no Portal de Periódicos da Coordenação de Aperfeiçoamento de Pessoal de Nível Superior (CAPES), que reúne as bases de dados SciELO, HighWire, Crossref, Scopus e Science Direct; no PubMed ${ }^{2}$, que incluí as bases de dados MEDLINE, OLDMEDLINE, Serials Database e NLM Catalog; e na Biblioteca Virtual em Saúde (BVS), também conhecida por Biblioteca Regional de Medicina (BIREME), que incluí as bases de dados LILACS, IBECS, MEDLINE, Biblioteca Cochrane e SciELO. Para a realização do referido levantamento, utilizou-se os descritores "Type 2 diabetes mellitus, chronic periodontitis, Biomarkers, Saliva". 


\section{RESULTADOS}

Atualmente há uma grande variedade de hipóteses etiológicas que têm sido propostas para explicar à patogênese e progressão da doença periodontal, contudo, todas têm o fator bacteriano como gatilho inicial. Esses, variam de específicas ou não específicas. Há a hipótese da placa, e, mais recentemente, a "polimicrobiana sinergia e disbiose"12,13. A maioria destas teorias Identificam as bactérias como a principal causa de patogênese ${ }^{13}$.

A prevalência de periodontite é significativamente maior entre as pessoas com diabetes mellitus mal controlados. A maioria da destruição tecidual na periodontite é considerada como o resultado de uma resposta inflamatória / imune excessiva à placa microbiana e envolve a libertação prolongada de espécies reativas de oxigénio (ROS). Há evidências de um aumento da capacidade antioxidante que compromete tecidos periodontais e fluidos que podem ser um fator adicional para danos nos tecidos na periodontite ${ }^{14}$.

A formação de ROS no biofilme da placa leva à destruição óssea alveolar e ao aprofundamento das bolsas periodontais. A inflamação periodontal resulta na liberação de citoquinas, prostaglandinas, toxinas bacterianas e enzimas de células hospedeiras e bacterianas, que têm efeitos prejudiciais adicionais nos tecidos ${ }^{15}$.

O diabetes mellitus (DM) é reconhecido como um importante fator de risco para periodontite, uma vez que os pacientes com DM apresentam maior prevalência e severidade das doenças periodontais, em comparação com aqueles sem $\mathrm{DM}^{16}$. Várias enzimas, proteínas e imunoglobulinas, que são constituintes abundantes da saliva, são biomarcadores potenciais para as doenças periodontais. As defensinas gengivais e as catelicidinas são pequenos peptídeos antimicrobianos catiônicos que desempenham um papel importante na resposta imunitária ${ }^{17}$.

Nas DP, tanto a resposta inata quanto a adaptativa dispõem de células de defesa que respondem à agressão tecidual. Na resposta inata existem duas grandes famílias de peptídeos antimicrobianos: as defensinas $(\alpha$ e $\beta)$ e as catelicidinas, onde já é evidenciado seus papeis nos agravos periodontais ${ }^{18}$. Os peptídeos identificados na cavidade oral são as $\beta$-defensinas, expressas pelo epitélio, $\alpha$-defensinas, pelos neutrófilos e o fragmento LL-37 (catelicidina), expresso tanto pelo epitélio quanto pelos neutrófilos, que, juntamente com a saliva, mantêm a saúde bucal ${ }^{19}$. A compreensão dos mecanismos imunológicos da patogênese da doença periodontal tem se tornado cada vez mais importante à medida que as evidências científicas indicam novas possibilidades de tratamento da doença ${ }^{20}$.

A IL-1 $\beta$, TNF- $\alpha$, IFN-y, IL-2 e IL-6 são citocinas que parecem ter papel importante na iniciação e progressão da periodontite. Os osteoclastos atingem os sítios de reabsorção via corrente sanguínea e o equilíbrio entre a deposição e a reabsorção de matriz óssea é regulado por parâmetros físicos (estímulos mecânicos, como a movimentação ortodôntica) e pela liberação de polipeptídeos (hormônios, como a PGE2 e o PTH, e citocinas, como a IL1, IL-6 e o TNF- $\alpha)^{20,21}$.

Todavia, nem todas as citocinas estão envolvidas com a destruição tecidual. No estudo de Sasaki et al. $(2008)^{22}$, por exemplo, o papel da interleucina 10 (IL-10) esteve relacionado com a proteção do hospedeiro frente à periodontite. Primeiramente, eles verificaram que camundongos com imunodeficiências de IL-10 exibiram alta suscetibilidade de DP. Depois, para estudarem o mecanismo de proteção por parte da IL-10, eles condi- 
cionaram alguns camundongos para exibir deficiência de uma molécula extremamente envolvida na sinalização da IL-10, a STAT3 ${ }^{18}$. No entanto, a interação de RANK/RANKL/ OPG é primordial no controle da remodelação óssea ${ }^{20}$.

O sistema receptor ativador do fator nuclear kappa ligante (RANKL)/RANK/ osteoprotegerina (OPG) atua em uma razão que determina a massa óssea e a integridade do esqueleto. Doenças periodontais que se relacionam à resposta imune do hospedeiro e à formação de lesões osteolíticas podem ser explicadas pela expressão diferencial destes fatores. A sinalização RANKL (expresso por osteoblastos ou células do estroma)/RANK regula a formação de osteoclastos multinucleados, bem como sua ativação e sobrevivência na remodelação óssea normal e em condições patológicas. De forma antagônica, OPG protege o esqueleto da reabsorção óssea excessiva, ligando-se a RANKL e evitando ligação ao receptor RANK. Em condições homeostáticas, RANKL e OPG apresentam níveis em equilíbrio de modo que não é limitado à osteoclastogênese. Com um estímulo inflamatório, a relação RANKL/OPG aumenta em tecidos periodontais e periapicais e eleva a estimulação da atividade dos osteoclastos e reabsorção óssea ${ }^{23}$.

A saliva é uma solução hipotônica formada pelo fluido crevicular gengival e exsudato da mucosa oral, é incolor, inodora e tem uma densidade relativa de 1,004-1,009 e um pH de 6,6 - 7,1. Aproximadamente $90 \%$ da saliva é secretada pelas glândulas salivares principais, glândulas parótidas, submandibulares e glândulas sublinguales. Sendo as com alta permeabilidade cercadas por abundantes capilares que podem trocar moléculas sanguíneas ${ }^{11}$. Os biomarcadores salivares já veem há algum tempo sendo utilizados na odontologia, como na detecção de câncer oral ${ }^{24}$, Síndrome de Sjogren ${ }^{25}$ e doenças periodontais ${ }^{26}$.

Porphyromonas gingivalis é uma bactéria que é intimamente associada à periodontite, sabendo disso, pesquisadores da University of Melbourne na Austrália desenvolveram um kit de biomarcadores salivares para essa bactéria, que é do complexo vermelho. Esse kit tem como base um ensaio imunoenzimático específico para a P. gingivalis. A tecnologia desenvolvida de diagnóstico é comparada com a reação em cadeia da polimerase em tempo real, altamente sensível, 92\%. Identifica estirpes isoladas em concentrações de $5 \times$ 104 a $5 \times 105 \mathrm{CFU} \cdot \mathrm{mL}-1^{26}$.

O estudo de Sanaka et al $(2017)^{5}$ identificou a cadaverina e hidrocinamato como marcadores salivares altamente específicos para a gravidade da inflamação periodontal. A produção de cadaverina é associada à putrefação e atribuída exclusivamente à descarboxilação bacteriana da lisina ${ }^{27}$. A relação do hidrocinamato, um análogo da fenilalanina, para a patogênese periodontal permanece amplamente desconhecido, embora um estudo anterior tenha mostrado que o hidrocinamato é produzido a partir de polifenóis pela microbiota intestina ${ }^{28}$, sugerindo sua origem microbiana.

Não obstante, outro estudo observou que os pacientes com periodontite exibiam níveis salivares aumentados de metabólitos de aminoácidos aromáticos ${ }^{29}$. Na patogênese da periodontite, os peptídeos antimicrobianos cooperam com outras proteínas inflamatórias e regulam vias inflamatórias distintas. Assim, uma abordagem combinatória, na qual os peptídeos antimicrobianos são medidos em conjunto com os seus ativadores ou proteínas alvos, aumentam o seu valor como biomarcadores de diagnóstico ${ }^{17}$.

Sanaka et al (2017) $)^{5}$ associa outros marcadores com a área de superfície inflamada periodontal (PISA), incluindo o ácido 5-aminovalérico, e a alanina_2TMS. Embora a asso- 
ciação de alanina_2TMS com uma maior PISA não seja clara, uma vez que diferenças na eficiência de derivatização pode influenciar nos achados. Ressalta-se que a abundância de aminoácidos descarboxilases na comunidade oral microbiana parece indicar um aumento das atividades relacionada à disbiose.

Outros biomarcadores detectados na saliva que refletem a condição sistêmica e oral do indivíduo que estão presentes em condições inflamatórias em indivíduos com DM2 e doença periodontal incluem interleucina 1- $\beta$ (IL-1 $\beta$ ) e proteína C-reativa (CRP) ${ }^{30}$. Também há uma interação positiva entre $\alpha$-2-macroglobulina e $\mathrm{HbA} 1 \mathrm{c}$, que evidencia os níveis de $\alpha$-2-macroglobulina na saliva como reflexo do controle glicêmico em pacientes com diabetes mellitus tipo $2^{31}$.

Constata-se, também, os níveis baixos de melatonina salivar em pacientes com diabetes tipo 2 e pacientes com periodontite. Sendo assim indica-se que a melatonina salivar tem um papel importante na patogênese da diabetes e das doenças periodontais e pode se tornar um biomarcador chave no diagnóstico e tratamento dessas duas doenças ${ }^{11,32}$.

\section{DISCUSSÃO}

Gurav e Jadhav (2011) em seu estudo evidencia a importância da correlação periodontite e diabetes, salienta que as profissões médicas e odontológicas devem trabalhar em conjunto. Os dentistas devem compreender os parâmetros da glicemia que são utilizados para estabelecer um diagnóstico de diabetes. Ao mesmo tempo, a periodontite, como uma infecção oral, permanece em grande parte não diagnosticada por médicos. Os sinais, sintomas e apresentação clínica da periodontite precisam ser reconhecidos pelos médicos para que os pacientes diabéticos sejam prontamente encaminhados para o tratamento, o que pode potencialmente prevenir complicações adicionais.

O estudo de Mesia et al (2016) ${ }^{33}$ tenta explicar o correção diabetes-periodontite, onde descreve-se uma resposta inflamatória induzida por polissacárideos (LPS) mais robusta, o que poderia explicar o aumento da susceptibilidade à doença periodontal em pacientes com DM2. Em apoio a isto, o seu estudo, também, observou uma correlação positiva e forte entre níveis estimulados por LPS de múltiplos marcadores inflamatórios, incluindo interleucina (IL)-6, IL-8 e MIP1 $\alpha$, com a profundidade da bolsa. Esta importante correlação foi observada somente dentro de Indivíduos com DM2, mas não com indivíduos sem diabetes. Esses dados reforçam a evidência de que uma resposta ao LPS por indivíduos com DM2 pode contribuir para a desagregação e / ou progressão exagerada da doença periodontal nesses pacientes com diabetes mellitus tipo 2.

Uma pessoa normal produz $600 \mathrm{~mL}$ de saliva por dia. A saliva consiste em $99 \%$ de água e o restante são moléculas orgânicas tais como amilase salivar, mucopolissacárido, mucina e lisozimas, e algumas matérias inorgânicas, tais como $\mathrm{Na}+, \mathrm{K}+, \mathrm{Ca} 2+$, $\mathrm{Cl}$ - e o ião tiocianato. A saliva tem múltiplos funções: ajuda na higiene da cavidade bucal, refresca a respiração, iniciadora da digestão a partir da amilase salivar que catalisa a hidrólise de amido em maltose e, por vezes, glicose na boca, bactericida tornando a saliva uma parte importante do sistema imunológico inespecífico humano ${ }^{11}$. É uma ferramenta de diagnóstico periodontal importante para detectar o início e progressão da doença, monitorizar a resposta à terapêutica ou medir o grau de susceptibilidade à progressão futura da doença que tem sido de grande interesse ao longo do tempo por se tratar alternativa auxiliadora não invasiva e rápida ${ }^{17}$. 
No estudo de Patel et al (2016) $)^{34}$, concluiu-se que biomarcadores como cálcio salivar, fósforo, fosfatase alcalina e $\mathrm{pH}$ podem ser considerados para avaliação do diagnóstico e prognóstico de doenças periodontais, sendo uma ferramenta útil para medir a eficácia da terapia, indo de encontro com os achados de Podzimek et al (2016) ${ }^{35}$, salienta que biomarcadores na saliva (por exemplo, enzimas, marcadores de proteínas ou stress oxidativo) podem ser utilizados para a determinação da atividade e para o prognóstico da doença periodontal. A saliva contém muitos marcadores que podem prever o risco de certas doenças (por exemplo, diabetes mellitus, cardiovasculares, oncológicas, endócrinas e doenças psiquiátricas).

Segundo Javaid et al (2016) $)^{30}$, a detecção precoce da doença não é apenas vital para reduzir a gravidade da doença e prevenir complicações, mas também crítico para aumentar a taxa de sucesso da terapia. Considerando a sua precisão, eficácia, facilidade de uso e custo-eficácia, os testes de diagnóstico salivar estarão disponíveis em consultórios odontológicos como opção possível em breve. Espera-se que o advento de ferramentas diagnósticas salivares sensíveis e específicas e o estabelecimento de diretrizes e resultados definidos após testes rigorosos permitirá que os mesmos sejam utilizados como testes rotineiros para várias doenças orais e sistêmicas no futuro.

\section{CONCLUSÃO}

A periodontite crônica é um sério problema de saúde pública no mundo. Uma vez associada ao DM2 torna-se ainda mais severa, tendo efeitos sistêmicos na piora do quadro clínico do paciente. Isso faz do diagnóstico precoce da periodontite crônica um aliado no aumento da qualidade de vida dos pacientes com DM2. Os métodos tradicionais de diagnóstico da periodontite crônica são invasivos e morosos o que torna ainda mais necessário a busca por alternativas clínicas satisfatórias. A utilização de biomarcadores na medicina é uma realidade difundida e tem relação direta com a modernização dos meios de saúde, na odontologia vem se pleiteando seu uso, mas ainda necessita de maiores estudos e aperfeiçoamentos das técnicas já empregadas com o intuito de diminuir seus custos, aumentar especificidade e acesso dos profissionais de saúde.

\section{REFERÊNCIAS BIBLIOGRÁFICAS}

1. Kassebaum NJ, Bernabé E, Dahiya M, Bhandari B, Murray CJ, Marcenes W. Global burden of severe periodontitis in 1990-2010: a systematic review and meta-regression. J Dent Res 2014; 93: 1045-1053.

2. Listl S, Galloway J, Mossey PA, Marcenes W. Global Economic Impact of Dental Diseases. J Dent Res 2015; 94: 1355-1361.

3. Dye BA. Global periodontal disease epidemiology. Periodontol 2000 2012; 58: 10-25.

4. Sanz M, Van Winkelhoff AJ. Periodontal infections: understanding the complexity--consensus of the Seventh European Workshop on Periodontology. J Clin Periodontol 2011; 38: 3-6.

5. Sakanaka A, Kuboniwa M, Hashino E, Bamba T, Fukusaki E, Amano A. Distinct signatures of dental plaque metabolic byproducts dictated by periodontal inflammatory status. Sci Rep 2017;7:42818.

6. Genco RJ, Borgnakke WS. Risk factors for periodontal disease. Periodontology 2000 2013; 62: 59-94.

7. Gurav A, Jadhav V. Periodontitis and risk of diabetes mellitus. J Diabetes. 2011; 3:21-8.

8. Miranda TS, Feres M, Retamal-Valdés B, Perez-Chaparro PJ, Maciel SS, Duarte PM. Influence of glycemic control on the levels of subgingival periodontal pathogens in patients with generalized chronic periodontitis and type 2 diabetes. J Appl Oral Sci 2017;25:82-89. 
9. Santos RC, Barros MSV, Pinho RCM, Cimões R. Saúde oral e qualidade de vida. Odontol Clín-Cient 2017; 16:15 - 18 .

10. Janem WF, Scannapieco FA, Sabharwal A, Tsompana M, Berman HA, Haase EM et al. Salivary inflammatory markers and microbiome in normoglycemic lean and obese children compared to obese children with type 2 diabetes. PLoS ONE 2017; 3: 0172647.

11. Zhang CZ, Cheng XQ, Li JY, Zhang P, Yi P, Xu X et al. Saliva in the diagnosis of diseases. International Journal of Oral Science 2016; 83:133-137.

12. Hajishengallis G, Lamont RJ. Beyond the red complexand into more complexity: the polymicrobial synergy and dysbiosis (PSD) model of periodontal disease etiology. Mol Oral Microbiol 2012; 27: 409-419.

13. Asad M, Abdul AAW, Raman RP, Harun HW, Ali TB, Chinna K et al. Comparison of nonsurgical periodontal therapy with oral hygiene instruction alone for chronic periodontitis. Journal of Oral Science 2017; 59:111-120.

14. Patil VS, Patil VP, Gokhale N, Acharya A, Kangokar P. Chronic Periodontitis in Type 2 Diabetes Mellitus: Oxidative Stress as a Common Factor in Periodontal Tissue Injury. Journal of Clinical and Diagnostic Research 2016; 10: 12-16

15. Soory M. Oxidative Stress Induced Mechanisms in the Progression of Periodontal Diseases and Cancer: A Common Approach to Redox Homeostasis? Cancers 2010;2:670-692.

16. Katagiri S, Nitta H, Nagasawa T, Izumi Y, Kanazawa M, Matsuo A et al. Effect of glycemic control on periodontitis in type 2 diabetic patients with periodontal disease. J Diabetes Investig 2013;4:320-325.

17. Güncü GN, Yilmaz D, Könönen E, Gürsoy UK. Salivary Antimicrobial Peptides in Early Detection of Periodontitis. Frontiers in Cellular and Infection Microbiology 2015;5:99.

18. Barbosa KGNB, Castro RD, Carvalho FG, Cabral YN. The Participation of immune responses in periodontal disease. Odontol Clín - Cient 2012;1:07-12.

19. Dale BA, Fredericks LP. Antimicrobial peptides in the oral environment: expression and function in health and disease. Curr Issues Mol 2005;7:119-34.

20. Rodrigues AZ, Kawata VKS , Júnior ABN , Sousa SLS , Grisi MFM , Palioto DB et al. Therapeutic strategies and potential targets for host response modulation of periodontal patients. R. Periodontia 2009; 19: 14-21.

21. Heymann D, Rousselle AV. gp130 cytokine family and bone cells. Cytokine 2000; 12: 1465-8.

22. Sasaki H, Suzuki N, Kent Jr. R, Kawashima N, Takeda J, Stashenko P. T Cell response mediated by myeloid cell-derived IL-12 Is responsible for porphyromonas gingivalis-induced periodontitis in IL-10-deficient mice. J Immun 2008;180:6193-98.

23. Cravo RX, Marinho CCB, Silva BVR, Silva BB, Oliveira PS, Silva IIC. Rankl/rank/opg: biomarcadores para estudos de metabolismo ósseo. In: XII Jornada de Odontologia da Universo; 2012. São Paulo. Anais. São Paulo: Revista de Trabalhos Acadêmicos; 2012.p. 58-58.

24. Wang Y, Springer S, Mulvey CL, Silliman N, Schaefer J, Sausen M. Detection of somatic mutations and HPV in the saliva and plasma of patients with head and neck squamous cell carcinomas. Sci Transl Med 2015; 7: 293-104.

25. Delaleu N, Mydel P, Kwee I, Brun JG, Jonsson MV, Jonsson R.High fidelity between saliva proteomics and thebiologic state of salivary glands defines biomarker signatures for primary Sjögren's syndrome. Arthritis Rheumatol 2015; 67: 1084-1095.

26. O'Brien-Simpson NM, Burgess K, Brammar GC et al. Development and evaluation of a saliva-based chairside diagnostic for the detection of Porphyromonas gingivalis. J Oral Microbiol 2015; 7: 29129.

27. Miller-Fleming L, Olin-Sandoval V, Campbell K, Ralser M. Remaining Mysteries of Molecular Biology: The Role of Polyaminesin the Cell. J Mol Biol 2015; 427: 3389-3406.

28. Ou K, Sarnoski P, Schneider KR, Song K, Khoo C, Gu L. Microbial catabolism of procyanidins by human gut microbiota. Mol Nutr Food Res 2014; 58: 2196-2205.

29. Barnes VM, Ciancio SG, Shibly O, Xu T, Devizio W, Trivedi HM et al. Metabolomics reveals elevated macromolecular degradation in periodontal disease. J Dent Res 2011;90: 1293-1297.

30. Javaid MA, Ahmed AS, Durand R, Tran SD. Saliva as a diagnostic tool for oral and systemic diseases. J Oral Biol Craniofac Res 2016;6: 66 - 75.

31. Aitken JP, Ortiz C, Morales-Bozo I, Rojas-Alcayaga G, Baeza M, Beltran C et al. $\alpha$-2-macroglobulin in saliva is associated with glycemic control in patients with type 2 diabetes mellitus. Dis Markers 2015; 89: 60-67.

32. Abdolsamadi H, Goodarzi MT, Ahmadi MF, Jazaeri M, Feradmal J, Zarabadi M et al. Reduction of melatonin level innpatients with type II diabetes and periodontal diseases. J Dent Res Dent Clin Dent Prospects 2014; 8: 160-165. 
33. Mesia R, Gholami F, Huang H, Clare-Salzler M, Aukhil I, Wallet SM et al. Systemic inflammatory responses in patients with type 2 diabetes with chronic periodontitis. BMJ Open Diabetes Research \& Care 2016;4: 000260.

34. Patel RM, Varma S, Suragimath G, Zope S. Estimation and Comparison of Salivary Calcium, Phosphorous, Alkaline Phosphatase and $\mathrm{pH}$ Levels in Periodontal Health and Disease: A Cross-sectional Biochemical Study. Journal of Clinical and Diagnostic Research 2016;7:58-61.

35. Podzimek S, Vondrackova L, Duskova J, Janatova T, Broukal Z. Salivary Markers for Periodontal and General Diseases. Disease Markers 2016; 482: 1122-1128 